\title{
Does therapeutic temperature management after cardiac arrest increase the risk of bleeding?
}

Williams TA, Mckenzie N, Inoue M.

Cardiac arrest is a significant public health issue, with a high case fatality ( $>90 \%$ overall). ${ }^{1}$

Guidelines recommend targeted temperature management (TTM) in the post-resuscitation care of patients who have suffered a cardiac arrest. ${ }^{2-5}$ Several studies ${ }^{6-8}$ have shown improved survival and neurological outcomes with the use of therapeutic hypothermia, also known as TTM. ${ }^{9}$ Possible complications of TTM include bleeding, shivering and infection. ${ }^{10-12}$ Hypothermia-induced coagulation disorder could enhance the risk for serious adverse events. ${ }^{5,12-14}$ but it is unclear if bleeding complications occur more often in patients suffering cardiac arrest who have TTM. This report critiques a systematic review and meta-analysis that examined the relationship of bleeding with TTM: Therapeutic temperature management after cardiac arrest and the risk of bleeding: Systematic review and meta-analysis for the effect of therapeutic temperature management after cardiac arrest. ${ }^{10}$

\section{Study summary}

Aim: The systematic review and meta-analysis aimed to examine whether TTM after cardiac arrest increased the risk of bleeding. Method: Data for the systematic review and meta-analysis were obtained by a systematic literature search in September 2013 using MEDLINE, EMBASE and CENTRAL databases. The level of evidence and quality were assessed using the International Liaison Committee on Resuscitation (ILCOR) Guidelines. The risk of bleeding while receiving TTM or no TTM was examined in two meta-analyses. Results: Of 941 studies, 34 met the selection criteria and were included in the systematic review. Bleeding was found to be higher in patients treated with TTM (RR: 1.30, 95\% Cl: 0.97-1.74) in a meta-analysis of 5 studies (599 patients) but this did not reach statistical significance $(p=0.085)$. In a second meta-analysis of bleeding requiring transfusion (7 studies, 599 patients), there was no significant difference in the incidence of severe bleeding (RR 0.97, 95\% Cl: 0.61-1.56, $\mathrm{p}=0.909$ ). Conclusion: The risk of bleeding was not significantly higher for patients undergoing TTM. Regarding the risk of bleeding, the authors asserted that TTM is a safe method for patients after cardiac arrest.

\section{Critique}


This report uses the PRISMA guidelines ${ }^{15}$ for the reporting of systematic reviews and meta-analysis to critique Stockman et al. ${ }^{10}$ The PRISMA guideline has 27 checklist items within seven Sections: Title, Abstract (Summary), Introduction, Methods, Results, Discussion and Funding.

The design 'Systematic review and meta-analysis' was included in the Title as required by PRISMA. The structured Summary included four sections: Aim, Methods, Results and Conclusions. The introductory comments in the Aim describe what is known about the topic of interest and what is unknown. The aim of the study should address the identified gap in knowledge but the specific aim was not explicit. The Methods listed three data sources used to identify relevant studies but important information such as study eligibility and data extraction were not provided. The quality of the included studies was assessed with the ILCOR Guidelines. ${ }^{16}$ Meta-analysis was used to synthesise the data for 12 studies. Results Thirty-four studies were included in the review. The two meta-analyses found no statistically significant difference for the risk of bleeding in TTM patients, either for bleeding of all severity (5 studies) or bleeding requiring transfusion (7 studies). The conclusions in the Summary and main text were different. There was insufficient evidence to support the authors' conclusions that TTM is a safe intervention for patients after cardiac arrest.

In the main text, the Introduction (which should include the rationale and aims of the study ${ }^{15}$ ) described the importance of TTM in the post-resuscitation care of cardiac arrest, the lack of information on complications with TTM and how this systematic review and meta-analysis would contribute to the gap in knowledge. There was no logical flow of concepts. The risks of TTM with particular attention to the risks of bleeding were described but a more succinct appraisal of the literature would have clarified the argument as to why patients may be at risk of bleeding after suffering a cardiac arrest.

The Methods used in the systematic review and meta-analysis provided insufficient information which potentially increased the risk of bias. The PRISMA guidelines include 12 items that should be described in the Methods. The authors did not indicate if a systematic review protocol existed. A prospectively pre-specified protocol minimises bias from selective reporting of results. ${ }^{17}$ Eligibility characteristics for adult studies where TTM was used in accordance with current guidelines were poorly described. Studies without a control group such as a study that compared two groups of hypothermic patients were excluded. Studies with a control group were included in the metaanalyses. Furthermore, information on eligibility criteria were distributed throughout several 
paragraphs in the Methods and Results. For example, "We did not use limitations concerning the time, the language, the study design or the study population at first"10, p1495 but non-English bibliographic databases were not included in the search strategy to search for non-English papers. In a later section of the paper studies were restricted to English, French and German languages with no rationale for the selection. Experimental studies were excluded but it is unclear as to whether this referred only to animal studies. Bleeding was grouped in five levels using the Bleeding Academic Research Consortium. ${ }^{18}$ Medical Subject Headings (MeSH) were used in the search strategy and further information was available in the online supplementary material. It is unknown if the authors attempted to obtain missing or unclear data from investigators to maximise data capture. Information was limited on how the data were collected and who collected the data. The use of one data collector limits interrater reliability but using two or more independent data collectors enables an objective assessment of the literature. Data were imported into standardised tables: this approach facilitates data consistency.

A description of the quality assessment was provided. Systematic reviews should include an assessment of study quality because their results are only as good as the quality of studies included. Studies were rated as good, fair or poor ${ }^{16}$ but it is unknown who performed the assessment. Only studies rated as good or fair quality were included in the systematic review and it is unknown if the cutpoint for inclusion was defined a priori. The risk of bias in individual studies was assessed using Cochrane Collaboration's tool ${ }^{19}$ and the results were reported in the online supplementary data. Interpretation of the results of a systematic review and meta-analysis relies on the risk of bias and the factors affecting the quality of evidence, such as imprecision, heterogeneity and publication bias. ${ }^{19}$ Visual inspection of funnel plots was performed to assess publication bias. Publication bias can occur when studies are more likely to be published because they report a significant treatment effect, are published in English, are frequently cited and have multiple publications compared to other studies. ${ }^{20}$

The methods of handling data and combining results of studies, including measures of consistency for each meta-analysis, were described. The meta-analyses used fixed effect models rather than a random effect model which was appropriate to the data because of the low heterogeneity. A sensitivity analysis of high and moderate-quality studies versus all studies was not performed although this should be done to test the robustness of the primary analysis. ${ }^{21}$ Forest plots that provide a graphical representation of the individual and pooled effect estimates were generated. 
The study selection was clearly shown in Figure 1 but the text description was confusing. Thirtyfour studies were included in the systematic review and 12 in the meta-analysis. The characteristics of included studies were described in Table 2. To improve clarity the description of study characteristics should be separated from the description of bleeding. Bleeding was higher in patients treated with TTM (RR: $1.30,95 \% \mathrm{Cl}: 0.97-1.74)$ but this did not reach statistical significance $(p=0.085)$. There was no significant difference in the incidence of severe bleeding requiring transfusion ( $\mathrm{RR} 0.97,95 \% \mathrm{Cl}$ : $0.61-1.56, \mathrm{p}=0.909$ ). The funnel plot for bleeding of all severity appeared balanced in relation to the small number of studies. In comparison the funnel plot of the bleeding requiring transfusion looked unbalanced, despite the slightly larger sample size. Excluding Holzer et al., ${ }^{11}$ the largest study with 1,038 patients, the sensitivity analysis of bleeding of all severity showed an increased risk of bleeding (RR 1.46, 95\% $\mathrm{Cl} 1.02-2.08, \mathrm{p}=$ 0.036).

The Discussion summarised the results of the systematic review and meta-analysis. Stockman et al. ${ }^{10}$ found bleeding was similar for patients who had TTM to those who did not. There are several studies that have reported the incidence of bleeding with TTM. ${ }^{11,14,22-25}$ The available evidence suggests bleeding is uncommon and severe bleeding is rare.

Stockman et al. ${ }^{10}$ acknowledge that there are limitations of the systematic review and metaanalysis. These included the inherent biases associated with systematic reviews and metaanalyses, e.g. identification of studies, selection of studies to be included (selection bias), publication bias. The description of the Methods did not provide sufficient information to discount the potential biases. Sample sizes for the meta-analyses were small and the meta-analyses may not have been sufficiently powered to show an association of bleeding on outcomes. The Conclusion in the abstract differs to the main text. There was insufficient evidence from this systematic review and meta-analysis to conclude that TTM is safe and the comment was not reported in the conclusion in the main text.

\section{Implications for practice}

Temperature management has been an important part of post-resuscitation care in patients who suffered a cardiac arrest since $2003 .^{2}$ The recommended temperature range is 32-34 degrees 
Celsius although the optimum temperature range is unknown. ${ }^{26}$ Nielsen ${ }^{27}$ compared a targeted temperature of 33 to 36 degrees Celsius in a randomised controlled trial and did not find a difference in outcomes but the authors acknowledged the importance of using some form of TTM.

Patients must not be allowed to get "hot", and this is especially important during the re-warming phase. The re-warming phase should be as slow and controlled as possible to prevent possible rebound hyperthermia, cerebral oedema and further brain injury. If the patient is allowed to get too "hot" the benefits of TTM may be limited. ${ }^{28,29} .{ }^{30} \mathrm{~A}$ target temperature of 36 to 37 degrees Celsius for 24 to 48 hours after reaching the re-warm target temperature and re-warming at a rate of 0.2 to 0.5 degrees Celsius per hour are suggested treatment regimens. ${ }^{14}$

Critical care nurses play a pivotal role in the initiation, maintenance and re-warming phases of TTM. Vigilance during TTM enables nurses to intervene early should there be a temperature excursion. Accurate documentation of TTM is imperative and includes the method of temperature control, mode of temperature measurement, start and end times for cooling and rewarming and the time target temperatures were reached. Monitoring the patient for complications is an important part of the care of these patients. Complications may occur at different phases of the intervention, for example bleeding may be more common during the cooling phase while allowing a patient to get hot during the rewarming phase is associated with poorer outcomes.

This systematic review and meta-analysis gives some insight into the risk of bleeding complications associated with TTM. Future research should include the optimum time of starting cooling, the duration of cooling, the optimal cooling method and the target temperature. ${ }^{31}$ Studies are also needed to improve our understanding of complications related to TTM.

\section{References}

1. Sasson C, Rogers MAM, Dahl J, Kellermann AL. Predictors of survival from out-of-hospital cardiac arrest: a systematic review and meta-analysis. Circ Cardiovasc Qual Outcomes 2010;3:6381.

2. Nolan JP, Morley PT, Hoek TLV, Hickey RW, Advancement Life support Task Force of the International Liaison committee on R. Therapeutic hypothermia after cardiac arrest. An advisory 
statement by the Advancement Life support Task Force of the International Liaison committee on Resuscitation. Resuscitation 2003;57:231-5.

3. Koster RW, Baubin MA, Bossaert LL, et al. European Resuscitation Council Guidelines for Resuscitation 2010 Section 2. Adult basic life support and use of automated external defibrillators. Resuscitation 2010;81:1277-92.

4. Neumar RW, Otto CW, Link MS, et al. Part 8: adult advanced cardiovascular life support: 2010 American Heart Association Guidelines for Cardiopulmonary Resuscitation and Emergency Cardiovascular Care. Circulation 2010;122:S729-67.

5. Deakin CD, Nolan JP, Soar J, et al. European Resuscitation Council Guidelines for Resuscitation 2010 Section 4. Adult advanced life support.[Erratum appears in Resuscitation. 2011 Jan;82(1):140]. Resuscitation 2010;81:1305-52.

6. Bernard SA, Gray TW, Buist MD, et al. Treatment of comatose survivors of out-of-hospital cardiac arrest with induced hypothermia. New England Journal of Medicine 2002;346:557-63. 7. Hypothermia after Cardiac Arrest Study Group. Mild therapeutic hypothermia to improve the neurologic outcome after cardiac arrest. N Engl J Med 2002;346:549-56.

8. Arrich J, Holzer M, Havel C, Mullner M, Herkner H. Hypothermia for neuroprotection in adults after cardiopulmonary resuscitation. Cochrane Database of Systematic Reviews 2012;9:CD004128.

9. Nunnally ME, Jaeschke R, Bellingan GJ, et al. Targeted temperature management in critical care: a report and recommendations from five professional societies. Crit Care Med 2011;39:111325.

10. Stockmann H, Krannich A, Schroeder T, Storm C. Therapeutic temperature management after cardiac arrest and the risk of bleeding: Systematic review and meta-analysis. Resuscitation $2014 ; 85$.

11. Holzer M, Mullner M, Sterz F, et al. Efficacy and safety of endovascular cooling after cardiac arrest: cohort study and Bayesian approach. Stroke 2006;37:1792-7.

12. Wolfrum S, Radke PW, Pischon T, Willich SN, Schunkert H, Kurowski V. Mild therapeutic hypothermia after cardiac arrest - a nationwide survey on the implementation of the ILCOR guidelines in German intensive care units. Resuscitation 2007;72:207-13.

13. Pell JP, Sirel JM, Marsden AK, Ford I, Walker NL, Cobbe SM. Presentation, management, and outcome of out of hospital cardiopulmonary arrest: comparison by underlying aetiology. Heart 2003;89:839-42. 
14. Polderman KH, Herold I. Therapeutic hypothermia and controlled normothermia in the intensive care unit: practical considerations, side effects, and cooling methods. Crit Care Med 2009;37:1101-20.

15. Moher D, Liberati A, Tetzlaff J, Altman DG, Group P. Preferred reporting items for systematic reviews and meta-analyses: the PRISMA statement. J Clin Epidemiol 2009;62:1006-12. 16. Morley P. Quality assessment for individual studies to be used for the review of the resuscitation science for 2010. http://www.heart.org/idc/groups/heartpublic/@wcm/@private/@ecc/documents/downloadable/ucm308201.pdf [accessed 23 Oct 2014]. 2008.

17. Finfer S, Bellomo R. Why publish statistical analysis plans? Crit Care Resuscitation 2009;11:5-6.

18. Mehran R, Rao SV, Bhatt DL, et al. Standardized bleeding definitions for cardiovascular clinical trials: a consensus report from the Bleeding Academic Research Consortium. Circulation 2011;123:2736-47.

19. Higgins J, Green S, (editors). Cochrane Handbook for Systematic Reviews of Interventions Version 5.1.0 [updated March 2011]. The Cochrane Collaboration, 2011. Available from www.cochrane-handbook.org. (accessed 29 Mar 2014). 2011.

20. Sterne JA, Egger M. Funnel plots for detecting bias in meta-analysis: guidelines on choice of axis. J Clin Epidemiol 2001;54:1046-55.

21. Muscedere J, Rewa O, McKechnie K, Jiang X, Laporta D, Heyland DK. Subglottic secretion drainage for the prevention of ventilator-associated pneumonia: A systematic review and metaanalysis. Crit Care Med 2011;39:1985-91.

22. Pittl U, Schratter A, Desch S, et al. Invasive versus non-invasive cooling after in- and out-ofhospital cardiac arrest: a randomized trial. Clin Res Cardiol 2013;102:607-14.

23. Arrich J, European Resuscitation Council Hypothermia After Cardiac Arrest Registry Study G. Clinical application of mild therapeutic hypothermia after cardiac arrest. Crit Care Med 2007;35:1041-7.

24. Schefold JC, Storm C, Joerres A, Hasper D. Mild therapeutic hypothermia after cardiac arrest and the risk of bleeding in patients with acute myocardial infarction. Int J Cardiol 2009;132:387-91.

25. Kozinski M, Pstragowski K, Kubica JM, et al. ACS network-based implementation of therapeutic hypothermia for the treatment of comatose out-of-hospital cardiac arrest survivors 
improves clinical outcomes: the first European experience. Scand J Trauma Resusc Emerg Med $2013 ; 21: 22$.

26. Australian Resuscitation Council. Therapeutic Hypothermia in Cardiac Arrest: An information update. Available from http://www.resus.org.au/files/arc therapeutic hypothermia.pdf Accessed 23 Oct 2014. 2012. 27. Nielsen N, Wetterslev J, Cronberg T, et al. Targeted Temperature Management at 33 degrees C versus 36 degrees C after Cardiac Arrest. N Engl J Med 2013.

28. Ferreira Da Silva IR, Frontera JA. Targeted temperature management in survivors of cardiac arrest. Cardiol Clin 2013;31:637-55, ix.

29. Kawahara F, Kadoi Y, Saito S, Goto F, Fujita N. Slow rewarming improves jugular venous oxygen saturation during rewarming. Acta Anaesthesiol Scand 2003;47:419-24.

30. Zeiner A, Holzer M, Sterz F, et al. Hyperthermia after cardiac arrest is associated with an unfavorable neurologic outcome. Arch Intern Med 2001;161:2007-12.

31. Nolan JP, Lyon RM, Sasson C, et al. Advances in the hospital management of patients following an out of hospital cardiac arrest. Heart 2012;98:1201-6. 